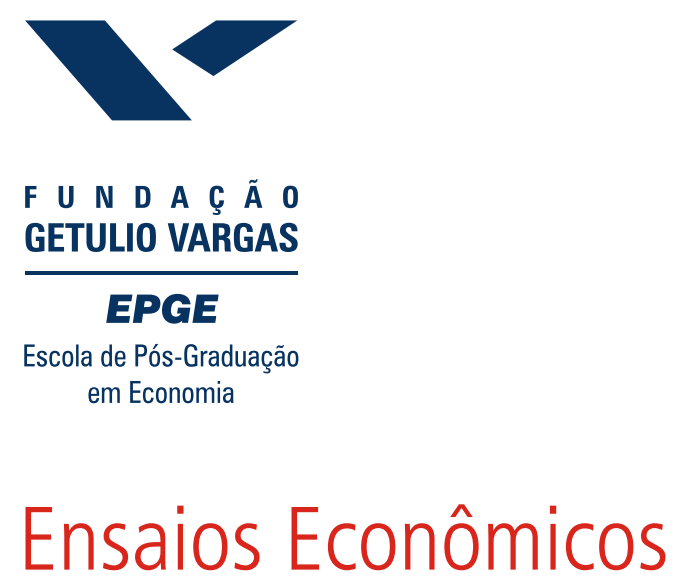

$$
\begin{aligned}
& \text { Escola de } \\
& \text { Pós Graduação } \\
& \text { em Economia } \\
& \text { da Fundação } \\
& \text { Getulio Vargas }
\end{aligned}
$$

Abstract types and distributions in independent private value auctions

Paulo Klinger Monteiro 
Os artigos publicados são de inteira responsabilidade de seus autores. As opiniões neles emitidas não exprimem, necessariamente, o ponto de vista da Fundação Getulio Vargas. 


\title{
Abstract types and distributions in independent private value auctions
}

\author{
Paulo Klinger Monteiro ${ }^{\star}$ \\ FGV-EPGE, Praia de Botafogo 190, sala 1103, 22250-900, RJ, Brazil
}

\begin{abstract}
In this note, in an independent private values auction framework, I discuss the relationship between the set of types and the distribution of types. I show that any set of types, finite dimensional or not, can be extended to a larger set of types preserving incentive compatibility constraints, expected revenue and bidder's expected utilities. Thus for example we may convexify a set of types making our model amenable to the large body of theory in economics and mathematics that relies on convexity assumptions. An interesting application of this extension procedure is to show that although revenue equivalence is not valid in general if the set of types is not convex these mechanism have underlying distinct allocation mechanism in the extension. Thus we recover in these situations the revenue equivalence.
\end{abstract}

JEL Classification: D44

Keywords: optimal auction, independent private values, revenue equivalence, mechanism design

\section{Introduction}

In this paper I study the relationship between types and distributions in an independent private values auction framework. The simplest assumption we can make on the set of possible types ${ }^{1}$ of an economic agent ${ }^{2}$ is that it is a finite set $T$. This however soon becomes unsatisfactory. Auction equilibria are in mixed strategies. We cannot use calculus, most distributions requires a continuum of types to exist and so on. The second simplest assumption we can make is that $T$ is a non-degenerated interval $[a, b] \subset \mathbb{R}$. Beyond this we may have multi-dimensional types $T=[a, b]^{l}$ or more complicated sets. I now focus on an independent private values auction

\footnotetext{
* Monteiro acknowledges the financial support of Edital Universal 02/06, CNPq.

1 Or valuations, signals.

2 I have in mind mainly a bidder in an auction setting.
} 
model with $I$ bidders. Thus suppose $T_{i}$, the set of types of Bidder $i=1, \ldots, I$, is given or appear endogenously in our model. Can we say something about the optimal auction? Can we talk about revenue equivalence if $T_{i}$ is not convex? I will show that if we have $\mathcal{M}=(q, P)$ a voluntary participation, incentive compatible mechanism defined on $T:=\prod_{i=1}^{I} T_{i}$ we can extend this mechanism, preserving voluntary participation and incentive compatibility constraints to a mechanism $\tilde{\mathcal{M}}$ defined on $\tilde{T}:=\prod_{i=1}^{I} \tilde{T}_{i} \supset T$. The only restriction we impose on $\tilde{T}_{i}$ is the minimal requirement of $\operatorname{Pr}\left(\tilde{T}_{i} \backslash T_{i}\right)=0$.

Suppose $T_{i} \subset \mathbb{R}$ is closed but is not an interval. How can we extend $T_{i}$ to $\tilde{T}_{i}=$ $\operatorname{con} T_{i}=\left[\min T_{i}, \sup T_{i}\right]$ ? Skreta (2006) present a method to extend an optimal mechanism $(q, P)$ defined on $T$ to $\tilde{T}$. To understand his extension note first that $\tilde{T}_{i} \backslash T_{i}$ is a countable union of disjoint intervals $I_{l}=\left(a_{l}, b_{l}\right)$. That is $\tilde{T}_{i} \backslash T_{i}=\cup_{l=1}^{\infty} I_{l}$. The extended mechanism is constant in $I_{l}$. This method works if we are looking for the optimal mechanism. In this case as Skreta shows a bidder with type $a_{l}<s<b_{l}$ chooses as type $a_{l}$. This method will not work if: (1) we allow for non-closed sets of types $^{3} ;(2)$ we want to extend mechanisms that are not optimal (like a first-price auction mechanism without a reserve price); (3) we have a higher dimensionality set of types. The extension procedure I will introduce allows for abstract set of types and any incentive compatible mechanism.

Once the extension procedure is accomplished we have very nice consequences. First we may search for the optimal auction in a classical setup. Thus suppose the set of types $x \in T_{i}$ has a distribution $F_{i}$ and this distribution has a density $f_{i}: T_{i} \rightarrow$ $(0, \infty)$. Extending $T_{i}$ to $\tilde{T}_{i}=\operatorname{con} T_{i}$ we still have a density $\tilde{f}_{i} ; \tilde{T}_{i} \rightarrow[0, \infty)$ but $\tilde{f}_{i}(x)=0$ in $\tilde{T}_{i} \backslash T_{i}$. Thus we would like to apply Myerson's (1981) characterization of the optimal auction. This is however not possible since a key hypothesis of Myerson (1981) is the strict positivity of the density on $\tilde{T}_{i}$. We may however apply Monteiro and Svaiter (2007) which characterizes the optimal auction for any distribution. A second consequence of the extension is that we recover revenue equivalence. For example if we initially have a two types set $T_{i}=\{a, b\}$ and two bidders. The optimal auction and the second price auction have the same allocation rule $q$ but do not have the same payment rule $P$. However if we extend the set of types to $\tilde{T}_{i}=[a, b]$ we see that the second-price auction and the optimal auction have distinct allocation rules which implies distinct payment rules.

A third consequence of the extension procedure is to make it clear that what matters is the distribution of types. The extension procedure adds a probability zero set of types. It makes it harder to satisfy incentive compatibility constraints. But those additional types should not matter. They do not change expected utilities nor expected revenue.

$\overline{3 \text { Like }}[0,1] \backslash \mathbb{Q}$. 


\section{Review of the auction model}

I first review the auction model with an abstract set of types. Let $\left(\Omega_{i}, \mathcal{B}_{i}\right)$ be a measurable space. Define $\mathcal{A}_{i}=\left\{B \cap S_{i} ; B \in \mathcal{B}_{i}\right\}$ and let $\left(S_{i}, \mathcal{A}_{i}, \mu_{i}\right)$ be a probability space, $i=1, \ldots, I$. We extend $\mu_{i}$ to $\mathcal{B}_{i}$ in a natural way: $\mu_{i}(B)=\mu_{i}\left(B \cap S_{i}\right)$. Thus $\mu_{i}\left(S_{i}^{c}\right)=0$. The valuation of Bidder $i$ with type $s \in S_{i}$ is given by $U^{i}(s)$ where $U^{i}: \Omega_{i} \rightarrow \mathbb{R}$ is a measurable function. Let $F_{i}: \mathbb{R} \rightarrow[0,1]$ be the distribution of $U^{i}$. Thus $F_{i}(x)=\mu_{i}\left(\left\{z \in S_{i} ; U^{i}(z) \leq x\right\}\right)$. Let $S:=\Pi_{i=1}^{I} S_{i}$ and $\mu=\mu_{1} \times \ldots \times \mu_{I}$ be the product probability. Thus the joint distribution is $F(s)=\prod_{i=1}^{I} F_{i}\left(s_{i}\right)$.

An object is to be sold at an auction with $I$ bidders. Each bidder knows his valuation $s_{i} \in S_{i}$. A direct mechanism is $(q, P)$ such that:

(1) $q=\left(q_{1}, \ldots, q_{I}\right), q_{i}: S \rightarrow[0,1]$;

(2) $\sum_{i=1}^{I} q_{i}(s) \leq 1$;

(3) $P=\left(P^{1}, \ldots, P^{I}\right), P^{i}(s) \in \mathbb{R}$;

(4) $\int\left|P^{i}\right| d \mu_{-i}<\infty$.

The auction proceeds as follows:

(i) Each bidder $i=1, \ldots, I$ announces privately and confidentially $s_{i} \in S_{i}$ to the seller. Let $s=\left(s_{1}, s_{2}, \ldots, s_{I}\right)$;

(ii) Bidder $i=1, \ldots, I$ pays $P^{i}(s)$;

(iii) Bidder $i$ receives the object with probability $q_{i}(s)$.

Define

$$
P_{i}\left(s_{i}\right):=\int P^{i}(s) d \mu_{-i}\left(s_{-i}\right) \text { and } Q_{i}\left(s_{i}\right):=\int q_{i}(s) d \mu_{-i}\left(s_{-i}\right) .
$$

The direct mechanism $(q, P)$ has to satisfy incentive compatibility (IC) and voluntary participations (VP) constraints. That is, for every $s_{i}, s_{i}^{\prime} \in S_{i}$,

$$
\begin{aligned}
& U^{i}\left(s_{i}\right) Q_{i}\left(s_{i}\right)-P_{i}\left(s_{i}\right)=\int\left(U^{i}\left(s_{i}\right) q_{i}(s)-P^{i}(s)\right) d \mu_{-i}\left(s_{-i}\right) \geq 0 \\
& U^{i}\left(s_{i}\right) Q_{i}\left(s_{i}\right)-P_{i}\left(s_{i}\right) \geq U^{i}\left(s_{i}\right) Q_{i}\left(s_{i}^{\prime}\right)-P_{i}\left(s_{i}^{\prime}\right) .
\end{aligned}
$$

Finally the seller's expected revenue is $\int \sum_{i} P^{i}(s) d \mu(s)$. I finish this section with a lemma that will be used in the next section.

Lemma 1 The expected payment $P_{i}(\cdot)$ is bounded from below.

Proof: Fix $a \in S_{i}$. The incentive compatibility constraint implies that for every $s \in$ $S_{i}, U^{i}(a) Q_{i}(a)-P_{i}(a) \geq U^{i}(a) Q_{i}(s)-P_{i}(s)$. Thus $P_{i}(s) \geq-\left|U^{i}(a)\right|+P_{i}(a)$ ending the proof. 


\subsection{The extension procedure}

Suppose $(q, P)$ is voluntary participation incentive compatible mechanism on $S$. We want to extend $(q, P)$ to an voluntary participation incentive compatible mechanism on $\Omega=\Omega_{1} \times \ldots \times \Omega_{I}$. Let $X_{i}=\left\{\left(Q_{i}(u), P_{i}(u)\right) ; u \in S_{i}\right\}$ and $A_{i}:=X_{i} \cup\{(0,0)\}$. Define $T_{i}: S_{i} \rightarrow \mathbb{R}$ the surplus: $T_{i}(s)=U^{i}(s) Q_{i}(s)-P_{i}(s)$. I begin with the following lemma.

Lemma 2 There exists a convex function $\phi_{i}: \mathbb{R} \rightarrow \mathbb{R}_{+}$such that $\phi_{i} \circ U^{i}=T_{i}$. And for every $s \in S_{i}$ it is true that $Q_{i}(s) \in \partial \phi_{i}\left(U^{i}(s)\right)$.

Proof: Define $\phi_{i}: \mathbb{R} \rightarrow \mathbb{R}$ by

$$
\phi_{i}(z)=\sup _{(Q, P) \in A_{i}} z Q-P .
$$

Lemma 1 implies that $\phi_{i}(z)$ is finite. And $(0,0) \in A_{i}$ implies $\phi_{i}(z) \geq 0$. Since $\phi_{i}$ is a supremum of linear functions, $z \rightarrow z \cdot Q-P$ it is a convex function. Fix $s \in S_{i}$ and $z^{\prime}:=U^{i}(s)$. The incentive compatibility constraints imply that $z^{\prime} Q_{i}(s)-P_{i}(s) \geq$ $z^{\prime} Q_{i}(x)-P_{i}(x)$ for every $x \in S_{i}$ and therefore $T_{i}(s)=z^{\prime} Q_{i}(s)-P_{i}(s)=\phi_{i}\left(U^{i}(s)\right)$. Note also that for every $s \in S_{i}$ we have that

$$
\phi_{i}(z)-\phi_{i}\left(U^{i}(s)\right) \geq z \cdot Q_{i}(s)-P_{i}(s)-\left(U^{i}(s) Q_{i}(s)-P_{i}(s)\right)=\left(z-U^{i}(s)\right) Q_{i}(s) .
$$

Thus $Q_{i}(s)$ is a subgradient of $\phi_{i}$. That is $Q_{i}(s) \in \partial \phi_{i}\left(U^{i}(s)\right)$.

QED

To define the extension I begin defining $\bar{Q}_{i}: \Omega_{i} \rightarrow[0,1]$ and $\bar{P}_{i}: \Omega_{i} \rightarrow \mathbb{R}:$

$$
\begin{aligned}
& \bar{Q}_{i}(\omega)=\left\{\begin{array}{cc}
Q_{i}(\omega) \quad \text { if } \quad \omega \in S_{i} ; \\
\left(\phi_{i}\right)_{+}^{\prime}\left(U^{i}(\omega)\right) \text { if } \omega \in \Omega_{i} \backslash S_{i} .
\end{array}\right. \\
& \bar{P}_{i}(\omega)=-\phi_{i}\left(U^{i}(\omega)\right)+U^{i}(\omega) \bar{Q}_{i}(\omega), \omega \in \Omega_{i} .
\end{aligned}
$$

I now define the probabilities $\bar{q}=\left(\bar{q}_{1}, \ldots, \bar{q}_{I}\right)$ for $\omega \in \Omega$. Let $\left\{e_{1}, e_{2}, \ldots, e_{I}\right\}$ be the canonical basis of $\mathbb{R}^{I}$. Thus $e_{1}=(1,0, \ldots, 0)$ and so on. For every $\omega \in \Omega$ let $I(\omega)=\left\{1 \leq j \leq I ; \omega_{j} \notin S_{j}\right\}$.

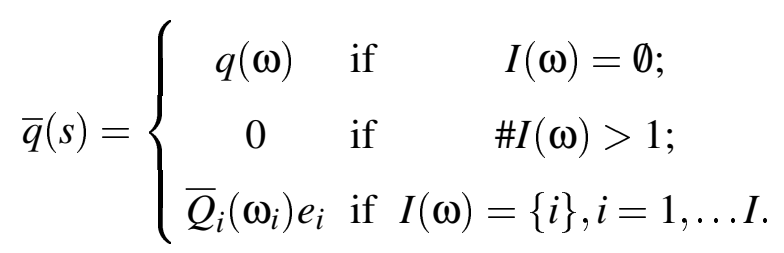

Thus the seller keeps the object if at least two bidders announces $\omega_{j} \notin S_{j}$. If only Bidder $i$ announces $\omega_{i} \notin S_{i}$ then $\bar{q}_{j}(\omega)=0$ for every $j \neq i$. And $\bar{q}_{i}(\omega)=\bar{Q}_{i}\left(\omega_{i}\right)$. 
Lemma 3 It is true that $\int_{\Omega_{-i}} \bar{q}_{i}(x, y) d \mu_{-i}(y)=\bar{Q}_{i}(x)$.

Proof: Let $l:=\int_{\Omega_{-i}} \bar{q}_{i}(x, y) d \mu_{-i}(y)$. First note that the integral over $\Omega_{-i} \backslash S_{-i}$ is null. If $x \in S_{i}$ then $\bar{q}_{i}(x, y)=q_{i}(x, y)$ for every $y \in S_{-i}$ and $l=\int_{\Omega_{-i}} q_{i}(x, y) d \mu_{-i}(y)=$ $Q_{i}(x)=\bar{Q}_{i}(x)$. If $x \notin S_{i}$ then $\bar{q}_{i}(x, y)=\bar{Q}_{i}(x)$ for every $y \in S_{-i}$ and $l=\bar{Q}_{i}(x)$ since $\mu_{-i}\left(S_{-i}\right)=1$.

QED

Theorem 1 The extended mechanism $(\bar{q}, \bar{P})$ satisfies voluntary participation and incentive compatibility constraints. It gives the same expected utility for Bidder $i$ if his type belongs to $S_{i}$ and gives the same revenue for the seller.

Proof: Let $T_{i}(\omega)=U^{i}(\omega) \bar{Q}_{i}(\omega)-\bar{P}_{i}(\omega)$ be type $\omega \in \Omega_{i}$ surplus. Suppose $\omega^{\prime} \in \Omega_{i}$. From definition (2) we get $T_{i}(\omega)=\phi_{i}\left(U^{i}(\omega)\right)$. Therefore $T_{i}(\omega) \geq 0$ and voluntary participation is satisfied. Incentive compatibility is also true:

$$
\begin{aligned}
& T_{i}(\omega) \geq \phi_{i}\left(U^{i}\left(\omega^{\prime}\right)\right)+\left(\phi_{i}\right)_{+}^{\prime}\left(U^{i}\left(\omega^{\prime}\right)\right)\left(U^{i}(\omega)-U^{i}\left(\omega^{\prime}\right)\right)= \\
& \quad T_{i}\left(\omega^{\prime}\right)+\bar{Q}_{i}\left(\omega^{\prime}\right)\left(U^{i}(\omega)-U^{i}\left(\omega^{\prime}\right)\right)=\bar{Q}_{i}\left(\omega^{\prime}\right) U^{i}(\omega)-\bar{P}_{i}\left(\omega^{\prime}\right) .
\end{aligned}
$$

Finally bidders' payoffs and seller's revenue are the same since $\mu_{-i}\left(S_{-i}^{c}\right)=0$.

Remark 1 It is clear from the construction that if $S_{i}^{\prime}$ is another set of types such that the distribution $F_{i}$ is the same then Bidder's expected utilities is the same and seller's revenue also is the same.

I now show how to make this extension in a simple example.

Example 1 Let $S_{i}=\{a, b\}, i=1,2$ where $0 \leq a<b$. Suppose that $\operatorname{Pr}(a)=\operatorname{Pr}(b)=$ $\frac{1}{2}$. Consider a second-price auction. That is $q_{1}(a, a)=\frac{1}{2}=q_{1}(b, b)$ and $q_{1}(a, b)=$ $0, q_{1}(b, a)=1$. The payment is $P^{1}\left(s_{1}, s_{2}\right)=\min \left\{s_{1}, s_{2}\right\}$. The expected payment of a type a bidder is $P_{1}(a)=P_{2}(a)=\frac{a}{4}$. The expected payment of type $b$ bidders $P_{1}(b)=P_{2}(b)=\frac{2 a+b}{4}$. The revenue is $R=\frac{3 a+b}{4}$. Let us extend the set of types to $\Omega_{i}=\mathbb{R}$ with valuation $U_{i}(t)=t$. The set $A_{i}=\left\{\left(\frac{1}{4}, \frac{a}{4}\right),\left(\frac{3}{4}, \frac{2 a+b}{4}\right),(0,0)\right\}$. The function $\phi_{1}(z)=\max \left\{0, \frac{z-a}{4}, \frac{3 z-2 a-b}{4}\right\}$. Thus

$$
\phi_{1}(z)=\left\{\begin{array}{c}
0 \quad \text { if } \quad z \leq a ; \\
\frac{z-a}{4} \quad \text { if } a \leq z \leq \frac{a+b}{2} ; \\
\frac{3 z-2 a-b}{4} \text { if } \quad \frac{a+b}{2} \leq z .
\end{array} \text { and } Q_{1}(z)=\left\{\begin{array}{c}
0 \text { if } \quad z \leq a ; \\
\frac{1}{4} \text { if } a<z \leq \frac{a+b}{2} \\
\frac{3}{4} \text { if } \quad \frac{a+b}{2}<z .
\end{array}\right.\right.
$$

In the optimal auction the allocation $q$ is the same as in the second price auction. However payment is different. The expected payment of type $a$ is $P_{1}(a)=\frac{a}{4}$. The expected payment of tybe $b$ is $P_{1}(b)=\frac{2 b+a}{4}$. Revenue is $\frac{b+a}{2}$. The probability of 
winning the object now is

$$
Q_{1}(z)=\left\{\begin{array}{l}
0 \text { if } \quad z<a ; \\
\frac{1}{4} \text { if } a \leq z<\frac{a+b}{2} ; \\
\frac{3}{4} \text { if } \quad \frac{a+b}{2} \leq z .
\end{array}\right.
$$

\section{Implications of the extension procedure for revenue equivalence}

In example 1 above we saw that distinct payment rules are possible for the same allocation rules if the set of types is not convex. I will show that if we enlarge the set of types (preserving the distribution as before) we may define an allocation rule which gives the right payoff. Thus by enlarging the set of types we may recover the revenue equivalence theorem. That is, the allocation rule for bidders that are "virtual" matters.

Theorem 2 Suppose $U^{i}\left(\Omega_{i}\right) \supset \operatorname{con} U^{i}\left(S_{i}\right)$. Then the payment $P_{i}$ is a function of $\bar{Q}_{i}$.

Proof: Let $\mathcal{S}=\{\{x\}, x \in \mathbb{R}\}$ be the set of singletons of real numbers. Let $e: \mathcal{S} \rightarrow \mathbb{R}$ be defined by $e(\{x\})=x$. For every $u \in U^{i}\left(\Omega_{i}\right)$ it is true that $\bar{Q}_{i}\left(\left(U^{i}\right)^{-1}(u)\right)=$ $\left\{\bar{Q}_{i}(\omega) ; U^{i}(\omega)=u\right\} \in \mathcal{S}$. Thus $\hat{Q}_{i}(u)=e\left(\bar{Q}_{i}\left(\left(U^{i}\right)^{-1}(u)\right)\right)$ is well defined. We have that

$$
\phi_{i}\left(U^{i}(\omega)\right)=\int_{-\infty}^{U^{i}(\omega)}\left(\phi_{i}\right)_{+}^{\prime}(u) d u=\int_{-\infty}^{U^{i}(\omega)} \hat{Q}_{i}(u) d u
$$

and therefore $\bar{P}_{i}(\omega)=U^{i}(\omega) \bar{Q}_{i}(\omega)-\int_{-\infty}^{U^{i}(\omega)} \hat{Q}_{i}(u) d u$.

\section{References}

[1] Skreta, V., 2006, Mechanism design for arbitrary type spaces, Economics Letters 91, 293-299;

[2] Monteiro, P. K. and B. F. Svaiter, 2007, Optimal auction with a general distribution: virtual valuation without a density, preprint

[3] Myerson, R. B., 1981, Optimal auction design, Mathematics of operations research, v.6 $n^{0} 1,58-73$; 